\title{
A Laboratory-Specific Scaling Factor to Predict the In Vivo Human Clearance of Aldehyde Oxidase Substrates ${ }^{\mathrm{E}}$
}

\author{
Mailys De Sousa Mendes, ${ }^{1}$ Alexandra L. Orton, Helen E. Humphries, Barry Jones, lain Gardner, \\ (1) Sibylle Neuhoff, and (1) Venkatesh Pilla Reddy ${ }^{1}$
}

Certara UK Limited, Simcyp Division, Sheffield, United Kingdom (M.D.S.M., H.E.H., I.G., S.N.) and Oncology DMPK Research \& Early Development (A.O., B.J.) and Modelling and Simulation, Research \& Early Development (V.P.R.), Oncology R\&D, AstraZeneca, Cambridge, United Kingdom

Received April 16, 2020; accepted July 22, 2020

\begin{abstract}
Aldehyde oxidase (AO) efficiently metabolizes a range of compounds with $\mathrm{N}$-containing heterocyclic aromatic rings and/or aldehydes. The limited knowledge of $\mathrm{AO}$ activity and abundance (in vitro and in vivo) has led to poor prediction of in vivo systemic clearance (CL) using in vitro-to-in vivo extrapolation approaches, which for drugs in development can lead to their discontinuation. We aimed to identify appropriate scaling factors to predict $A O C L$ of future new chemical entities (NCEs). The metabolism of six AO substrates was measured in human liver cytosol (HLC) and S9 fractions. Measured blood-toplasma ratios and free fractions (in the in vitro system and in plasma) were used to develop physiologically based pharmacokinetic models for each compound. The impact of extrahepatic metabolism was explored, and the intrinsic clearance required to recover in vivo profiles was estimated and compared with in vitro measurements. Using HLC data and assuming only hepatic metabolism, a systematic underprediction of clearance was observed (average fold underprediction was 3.8). Adding extrahepatic metabolism improved the
\end{abstract}

accuracy of the results (average fold error of 1.9). A workflow for predicting metabolism of an NCE by AO is proposed, and an empirical (laboratory-specific) scaling factor of three on the predicted intravenous CL allows a reasonable prediction of the available clinical data. Alternatively, considering also extrahepatic metabolism, an scaling factor of 6.5 applied on the intrinsic clearance could be used. Future research should focus on the impact of the in vitro study designs and the contribution of extrahepatic metabolism to AO-mediated clearance to understand the mechanisms behind the systematic underprediction.

\section{SIGNIFICANCE STATEMENT}

This works describes the development of scaling factors to allow in vitro-in vivo extrapolation of the clearance of compounds by aldehyde oxidase metabolism in humans. In addition, physiologically based pharmacokinetic models were developed for each of the aldehyde oxidase substrate compounds investigated.

\section{Introduction}

Aldehyde oxidase (AO) is a cytosolic molybdenum-containing enzyme that very efficiently oxidizes a range of N-containing heterocyclic aromatic rings and aldehydes (Montefiori et al., 2017). The limited knowledge about AO activity, abundance, and translation from in vitro to in vivo has led to poor prediction of in vivo clearance (CL) and consequently to clinical failure of some AO substrates (Fan et al., 2016; Jensen et al., 2017). The reasons for the poor prediction of CL of aldehyde oxidase substrates $\left(\mathrm{CL}_{\mathrm{AO}}\right)$ are multiple: Firstly, $\mathrm{AO}$ is only expressed in the cytosol, and therefore standard metabolism studies

No funding was received for this work.

${ }^{1}$ M.D.S.M. and V.P.R. contributed equally as joint first authors.

V.P.R., A.O., and B.J. are full-time employees of AstraZeneca when this study was conducted and hold shares of AstraZeneca. M.D.S.M., H.E.H., I.G., and S.N. employees of Certara UK Limited when this study was conducted.

https://doi.org/10.1124/dmd.120.000082.

S This article has supplemental material available at dmd.aspetjournals.org. using human liver microsomes (HLMs) will overlook AO metabolism (Obach, 2011; Zientek and Youdim, 2015; Dalvie and Di, 2019). Secondly, scaling from animal data can be challenging because dogs have low $\mathrm{AO}$ expression, and the $\mathrm{AO}$ expression in different rat strains is very variable (Dalvie et al., 2013; Tanoue et al., 2013). Additionally, although monkey, rat, and rabbit $\mathrm{AO}$ have been investigated, the metabolism of compounds by AO in these species only moderately overlaps with human clearance by AO (Al salhen, 2014; Dick, 2018). Finally, the interindividual variability in AO expression in humans is high, and consequently, the risk of having data for a nonrepresentative individual is considerable (Hutzler et al., 2014). In 2010, Zientek et al. (2010) have predicted the in vivo intrinsic clearance $\left(\mathrm{CL}_{\mathrm{int}}\right)$ for $\mathrm{AO}$ from in vitro data and compared them to the $\mathrm{CL}_{\mathrm{int}, \mathrm{AO}}$ estimated after intravenous administration for five compounds. The $\mathrm{CL}_{\mathrm{int}, \mathrm{AO}}$ was underestimated by 13-fold (range: 5-32) when using human liver cytosol (HLC) data and by 15-fold (range: 3-52) when using human liver S9 (HLS9) data. Strategies have been formulated to handle AOmediated clearance in drug discovery and development. However, understanding why human in vivo clearance is underpredicted using

ABBREVIATIONS: $\mathrm{ACN}$, acetonitrile; $\mathrm{AO}$, aldehyde oxidase; $\mathrm{AUC}$, area under the curve; $\mathrm{B} / \mathrm{P}$, blood/plasma ratio; $\mathrm{CL}_{\text {, clearance; } \mathrm{CL}} \mathrm{AO}, \mathrm{CL}$ of aldehyde oxidase substrates; $\mathrm{CL}_{\text {int }}$, intrinsic $\mathrm{CL} ; \mathrm{CL}_{\mathrm{IV}}$, intravenous $\mathrm{CL} ; \mathrm{fm}$, fraction metabolized; fu, unbound fraction in plasma; $\mathrm{HLC}$, human liver cytosol; HLM, human liver microsome; HLS9, human liver S9; HPLC, high-pressure liquid chromatography; IVIVE, in vitro-to-in vivo extrapolation; LC-MS/MS, liquid chromatography-tandem mass spectrometry; NCE, new chemical entity; PBPK, physiologically based pharmacokinetic; PK, pharmacokinetics; prop, proportion. 
TABLE 1

PBPK model input parameters and clinical data used for PBPK model verification. AGP, Alpha 1 Acid Glycoprotein; LOQ, limit of quantifiation; MW, molecular weight;

Phys Chem, physicochemical properties; pka, negative log of the acid dissociation constant.

When several data were available, a weighted mean was calculated.

\begin{tabular}{|c|c|c|c|c|c|c|c|}
\hline \multicolumn{2}{|c|}{ Compound } & $\mathrm{O}^{6}$-Benzylguanine & BIBX1382 & Carbazeran & Zaleplon & Ziprasidone & Zoniporide \\
\hline \multicolumn{8}{|l|}{ Phys Chem } \\
\hline \multicolumn{2}{|l|}{$\mathrm{MW}(\mathrm{g} / \mathrm{mol})$} & 241.25 & 387.84 & 360.41 & 305.33 & 412.94 & 320.35 \\
\hline \multicolumn{2}{|l|}{$\log \mathrm{P}$} & 1.04 (Liu et al., 2005) & $3.97^{a}$ & $1.83^{a}$ & $1.3^{a}$ & $4.53^{a}$ & $1.15^{a}$ \\
\hline \multicolumn{2}{|c|}{ Compound type } & Ampholyte & Diprotic base & Base & Neutral & Diprotic base & Diprotic base \\
\hline \multicolumn{2}{|l|}{ pka } & $9.35 ; 3.361^{a}$ & $2.83 ; 8.64^{a}$ & $8.6^{a}$ & - & $6.31 ; 8.24^{a}$ & $\begin{array}{l}3.4 ; 7.2 \text { (Tracey } \\
\text { et al., 2003) }\end{array}$ \\
\hline \multicolumn{2}{|l|}{$\mathrm{B} / \mathrm{P}$} & $0.9^{a}$ & $1.45^{a}$ & $0.735^{a}$ & 0.853 & $0.63^{a}$ & $0.938^{a}$ \\
\hline \multicolumn{2}{|l|}{ fu } & $0.14^{a}$ & $0.12^{a}$ & $0.09^{a}$ & $0.576^{a}$ & $0.001^{a}$ & $0.421^{a}$ \\
\hline \multicolumn{2}{|c|}{ Main binding protein } & $\mathrm{HSA}^{b}$ & $\mathrm{AGP}^{b}$ & $\mathrm{AGP}^{b}$ & $\mathrm{HSA}^{b}$ & $\mathrm{AGP}^{b}$ & $\mathrm{AGP}^{b}$ \\
\hline \multicolumn{8}{|l|}{ Elimination } \\
\hline \multicolumn{2}{|c|}{ 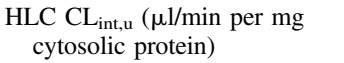 } & $10.9^{a}$ & $1062^{a}$ & $175^{a}$ & $1.86^{a}$ & $33.4^{a}$ & $13.1^{a}$ \\
\hline \multicolumn{2}{|c|}{$\begin{array}{l}\text { HLS9-NADPH } \mathrm{CL}_{\text {int,u }} \\
\quad(\mu 1 / \text { min per mg S9 protein })\end{array}$} & $2.2^{a}$ & $177.3^{a}$ & $72^{a}$ & $<1^{a}$ & $150.6^{a}$ & $2.8^{a}$ \\
\hline \multicolumn{2}{|c|}{$\begin{array}{l}\mathrm{HLM}_{, \mathrm{u}}(\mu \mathrm{l} / \mathrm{min} \text { per } \mathrm{mg} \\
\text { microsomal protein })\end{array}$} & $7.9^{a}$ & $47.3^{a}$ & $6.7^{a}$ & $\begin{array}{l}3.06 \text { (Renwick } \\
\text { et al., 2002) }\end{array}$ & $587.8^{a}$ & $<\mathrm{LOQ}^{a}$ \\
\hline \multicolumn{2}{|c|}{ Renal clearance $(1 / \mathrm{h})$} & $\begin{array}{l}0.12 \text { (Dolan et al., 1998; } \\
\text { Tserng et al., 2003) }\end{array}$ & $\begin{array}{l}1.94 \text { (Hutzler } \\
\text { et al., 2012) }\end{array}$ & $\begin{array}{l}0 \text { (Kaye et al., } \\
\text { 1984) }\end{array}$ & - & $\begin{array}{l}1.65 \text { (Miceli et al., } \\
\text { 2005) }\end{array}$ & $\begin{array}{c}16.4 \text { (Dalvie et al., } \\
\text { 2013) }\end{array}$ \\
\hline \multicolumn{2}{|c|}{ Biliary CL (1/h) } & - & - & - & - & - & $\begin{array}{c}0.96 \text { (Dalvie et al., } \\
\text { 2013) }\end{array}$ \\
\hline \multirow{2}{*}{\multicolumn{2}{|c|}{$\begin{array}{l}\text { Predicted } \mathrm{fm}_{\mathrm{AO}} \\
\text { Observed in vivo } \mathrm{CL}\end{array}$}} & 0.75 & 0.97 & 0.98 & 0.55 & 0.064 & 0.56 \\
\hline & & & & & & & \\
\hline \multicolumn{2}{|c|}{$\mathrm{CL}_{\mathrm{IV}}(\mathrm{l} / \mathrm{h})$ (plasma) } & $\begin{array}{c}61.70 \pm 22.14 \text { (Dolan et al., } \\
1998 ; \text { Tserng et al., 2003) }\end{array}$ & $\begin{array}{c}161.50 \pm 42 \\
\text { (Hutzler et al., } \\
\text { 2012) }\end{array}$ & $\begin{array}{c}157.92 \pm 34.86 \\
\text { (Kaye et al., 1984) }\end{array}$ & $\begin{array}{c}61.48 \pm 14.53 \\
\text { (Rosen et al., 1999) }\end{array}$ & $\begin{array}{c}22.50 \pm 3.15 \\
\text { (Miceli et al., } \\
\text { 2005) }\end{array}$ & $\begin{array}{c}96.39 \pm 5.51 \\
\text { (Dalvie et al., } \\
2013)\end{array}$ \\
\hline \multirow{5}{*}{$\begin{array}{l}\text { Population } \\
\text { Simulation } \\
\text { settings }\end{array}$} & & Sim-Cance & & & Sim-Healthy & volunteers & \\
\hline & $\begin{array}{l}\text { Number of } \\
\text { trials }^{c}\end{array}$ & 20 & 10 & 10 & 10 & 10 & 20 \\
\hline & Subject/trial & 6 & 11 & 10 & 10 & 13 & 4 \\
\hline & Age range (yr) & $32-74$ & $50-73$ & $20-50$ & $19-32$ & $19-37$ & $18-55$ \\
\hline & Prop. female & 0.3 & 0.63 & 0.5 & 0.5 & 0 & 0 \\
\hline
\end{tabular}

${ }^{a}$ In-house data.

${ }^{b}$ See text.

${ }^{c}$ To ensure that the simulated population will be representative of the global population, the number of trials was increased when the number of subjects per trial was low.

in vitro $\mathrm{CL}_{\mathrm{int}, \mathrm{AO}}$ from HLC and HLS9 is necessary to understand the role of $\mathrm{AO}$ in the metabolism of new chemical entities in a wider chemical space.

In this work, we aimed 1) to assess the prediction of intravenous clearance $\left(\mathrm{CL}_{\mathrm{IV}}\right)$ of six $\mathrm{AO}$ substrates from in vitro data and then 2) to derive an empirical scaling factor that 3 ) could be used to predict the $\mathrm{CL}$ of future NCE using physiologically based pharmacokinetic (PBPK) modeling. Initially, the metabolism by AO was assumed to occur only in the liver, and results from HLC and HLS9 were compared. However, there is compelling evidence of extrahepatic metabolism ${ }^{\left(\mathrm{CL}_{\mathrm{IV}}\right.}$ greater than the hepatic blood flow and AO expression data in extrahepatic tissues); hence, the impact of extrahepatic metabolism was also explored. Finally, scaling factors were estimated to optimally recover in vivo concentration-time profiles.

\section{Materials and Methods}

Pooled (150 donors; lot 38289) human liver cytosol, pooled (150 donors; lot 3829) human liver S9, and pooled (150 donors; equal sex mix) human liver microsomes were obtained from Corning Life Sciences (Woburn, MA). It is possible that the human donor liver tissues might have been perfused or preserved with University of Wisconsin solution or another allopurinol-containing buffer, which may exhibit aldehyde oxidase inhibition potential at high concentrations.

Frozen human plasma (pooled from 78 individuals, mixed sex) generated using K2-EDTA as an anticoagulant was purchased from BioreclamationIVT (Baltimore, $\mathrm{MD}$ ). AO substrates $\mathrm{O}^{6}$-benzylguanine, zaleplon, zoniporide, and carbazeran were sourced from Sigma-Aldrich (Poole, UK). BIBX1382 was sourced from Santa Cruz Biotechnology (Dallas, TX). Ziprasidone was synthesized at AstraZeneca (Cambridge, UK). Formic acid, ammonium formate, and DMSO were purchased from Sigma-Aldrich. HPLC-grade methanol, water, and acetonitrile (ACN) were obtained from Thermo Fisher Scientific (Waltham, MA). All other solvents were HPLC-grade and, unless otherwise specified, all other reagents were purchased from Sigma-Aldrich.

Compound Selection. The aim of this work was to assess the robustness of the prediction of $\mathrm{CL}_{\mathrm{AO}}$. To remove the additional uncertainty associated with predicting processes influencing oral bioavailability, only drugs with reported intravenous clearance and a fraction metabolized $(\mathrm{fm})$ by $\mathrm{AO}\left(\mathrm{fm}_{\mathrm{AO}}\right)$ of greater than $5 \%$ were selected for inclusion in this exercise. Using these criteria, the involvement of $\mathrm{AO}$ in the metabolism of $\mathrm{O}^{6}$-benzylguanine, BIBX1382, carbazeran, zaleplon, ziprasidone, and zoniporide was investigated in this study (Figure 1). Physicochemical data, including molecular weight, $\log \mathrm{P}$ and $\mathrm{pKa}$, $\mathrm{acid} / \mathrm{base}$ nature as well as blood binding properties, and information about the compound elimination, were compiled for all drugs (Table 1). When data from several reliable sources were available, a weighted mean value was used.

Determination of Aldehyde Oxidase Metabolic $\mathbf{C} \mathbf{L}_{\text {int }}$. The AO-mediated metabolism was measured in incubations containing either an HLC suspension at $1 \mathrm{mg}$ protein/ml or HLS9 suspension at $2.5 \mathrm{mg}$ protein $/ \mathrm{ml}$, both in phosphate buffer $(100 \mathrm{mM}), \mathrm{pH}$ 7.4. The reactions were initiated by addition of prediluted compounds $(2.5 \mu \mathrm{l}$ from $100 \mu \mathrm{M}$ in $100 \mathrm{mM}$ Phosphate buffer/ACN/DMSO 90/9/1) to give a final nominal concentration of $1 \mu \mathrm{M}$. The solvent concentration did not exceed a total of $0.1 \%$. The samples were then incubated at $37^{\circ} \mathrm{C}$ for either 120 minutes in HLC or 60 minutes in HLS9, with time points taken at 10, 30, 60, 90, and 120 minutes and 5, 10, 20, 40, and 60 minutes, respectively. The aliquots $(25 \mu \mathrm{l})$ were precipitated with $\mathrm{ACN}(1$ in $5 \mathrm{v} / \mathrm{v})$ containing internal standard (historic AstraZeneca compound; AZ10024306) and centrifuged at $3500 \mathrm{rpm}$ for 10 minutes, and the supernatant was diluted 1 in $7(\mathrm{v} / \mathrm{v})$ with ultra-pure HPLC water before analysis by liquid chromatography-tandem mass spectrometry (LC-MS/MS). All incubations were carried out in duplicate. The in vitro elimination rate constant corresponding to parent compound depletion was determined for each reaction using the first-order decay calculation in Microsoft Excel Sheet. 
Determination of Unbound Fraction in Human Plasma. The extent of binding of compounds to plasma proteins was determined by equilibrium dialysis at a compound concentration of $5 \mu \mathrm{M}$ using the Rapid Equilibrium Device (Thermoscientific Pierce). Phosphate buffer (100 mM, pH 7.4) was added to the buffer chamber, and $300 \mu$ l of plasma was spiked with compound to the sample chamber. The unit was covered with a gas-permeable lid and incubated for 18 hours at $37^{\circ} \mathrm{C}$ at $300 \mathrm{rpm}$ with $5 \% \mathrm{CO}_{2}$. At the end of incubation, samples $(50 \mu \mathrm{l})$ from both buffer and plasma chambers were removed for analysis. Samples and standards were matrix-matched and analyzed using LC-MS/MS. The unbound fraction in plasma (fu) was calculated as follows:

$$
f u=\frac{\text { Concentration in buffer chamber }}{\text { Concentration in plasma chamber }}
$$

Determination of Blood-to-Plasma Ratio. A volume of plasma sufficient for the assay was obtained from whole human blood by centrifugation $(3220 \mathrm{~g}$ for 10 minutes at $\left.4^{\circ} \mathrm{C}\right)$. The test compound $(10 \mu \mathrm{M})$ was added to $398 \mu \mathrm{l}$ of the prewarmed human plasma and blood separately and incubated for 30 minutes. After incubation, the blood samples were centrifuged for 10 minutes at $3220 \mathrm{~g}$ $\left(37^{\circ} \mathrm{C}\right)$, and the plasma samples were stored at $37^{\circ} \mathrm{C}$. Aliquots $(400 \mu \mathrm{l})$ of ice-cold acetonitrile containing internal standard were added to $100-\mu 1$ samples of plasma separated from centrifuged whole blood and to reference plasma samples. These samples were then centrifuged, diluted with distilled water, and analyzed by LCMS/MS to determine the compound concentration. Blood/plasma ratio (B/P) was calculated as follows:

$$
\mathrm{B} / \mathrm{P}=\frac{\text { Concentration in reference plasma }}{\text { Concentration in plasma from blood }}
$$

Determination of Unbound Fraction in HLM. The extent of binding of compounds to HLM was determined by equilibrium dialysis using the HT Dialysis LLC device (Gales Ferry, CT) with HLM at a concentration of $1 \mathrm{mg}$ protein $/ \mathrm{ml}$ and a final compound concentration of $1 \mu \mathrm{M}$. PBS $(150 \mu \mathrm{l})$ was added to the buffer well and $150 \mu 1$ HLM containing the compound to the sample well and incubated at $37^{\circ} \mathrm{C}$ for 4 hours. After the incubation, $50-\mu l$ aliquots from both donor and receiver wells were removed for analysis. Samples and standards were matrix-matched and analyzed by LC-MS/MS. The unbound fraction in the incubation $\left(\mathrm{fu}_{\mathrm{mic}}\right)$ was calculated as follows:

$$
F u_{m i c}=\frac{\text { Conc in buffer well }}{\text { Conc in microsmal suspension well }}
$$

LC-MS/MS Analysis. The concentration of all compounds in the incubations was determined by LC-MS/MS. An Acquity ultra-performance liquid chromatography system (Waters, UK) coupled to a triple-quadrupole mass spectrometer (Xevo TQ-S; Waters, Milford, MA) was used to carry out the sample analysis. The details of quantification of analytes are described in Supplemental text. Detection of the ions was performed in the multiple reaction monitoring mode. Peak integration and calibrations were performed using TargetLynx software (Version 4.1; Waters).

Prediction of Intravenous Clearance Using PBPK Models. The clinical trials providing the reference $\mathrm{CL}_{\mathrm{IV}}$ have been conducted in subjects with variable demographic characteristics (i.e., age range, proportion of females, healthy/patients with cancer). The specific demographics will influence some of the physiologic parameters (i.e., liver weight, plasma protein concentration) that in turn can impact the pharmokinetics (PK) parameters observed. Therefore, PBPK models were developed for each drug using the Simcyp Simulator V18R2, and the simulated trial designs and virtual population were selected accordingly to match the observed clinical trial (Table 1) (Kaye et al., 1984; Dolan et al., 1998; Rosen et al., 1999; Tserng et al., 2003; Miceli et al., 2005; Hutzler et al., 2012; Dalvie et al., 2013).

The $\mathrm{CL}_{\text {int }}$ obtained in vitro from HLC and HLS9 fractions was corrected by the free fraction in the in vitro assay. The free fraction in HLM was measured using $1 \mathrm{mg} / \mathrm{ml}$ of microsomal protein. No clear trend concerning the difference in binding between HLC and HLM was observed, and therefore, the binding was assumed to stay the same in HLC and HLS9 fraction (Cubitt, 2009). When the protein concentrations used were different from the $1 \mathrm{mg} / \mathrm{ml}$ assessed in the binding experiments, the free fraction was extrapolated using the equation from Austin et al. (2002)<smiles>Nc1nc(COc2ccccc2)c2ncnc-2[nH]1</smiles>

O6-benzylguanine<smiles>CCN(C(C)=O)c1cccc(-c2ccnc3c(C#N)cnn23)c1</smiles>

Zaleplon<smiles>CN1CCC(Nc2ncc3ncnc(Nc4ccc(F)c(Cl)c4)c3n2)CC1</smiles>

BIBX-1382<smiles></smiles>

Ziprasidone (AO cleaves bond)<smiles>CCNC(=O)OC1CCN(c2nnc(C#N)cc2-c2cc(OC)c(OC)cc2Cl)CC1</smiles><smiles>N=C(N)NC(=O)c1cnn(-c2cccc3ncccc23)c1C1CC1</smiles>

Zoniporide

Fig. 1. Chemical structure of aldehyde oxidase substrate that have reported intravenous clearance. 


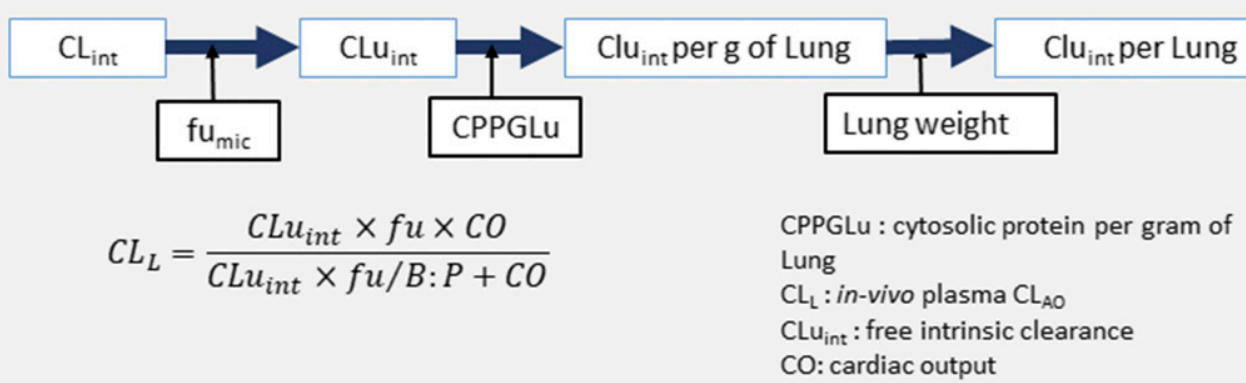

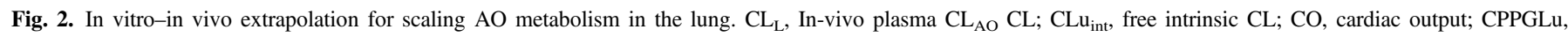
cytosolic protein per gram of lung.

The $\mathrm{B} / \mathrm{P}$, fu and $\mathrm{fu}_{\mathrm{mic}}$, and in vitro metabolism data were used to develop PBPK models for each compound (Table 1). The main plasma-binding protein was assumed to be albumin for the acid, neutral, and ampholyte compounds and $\alpha-1$ acid glycoprotein for the basic compounds. Physicochemical properties were gathered from literature sources and whole-body PBPK models with predicted volumes of distribution calculated using the Rodgers and Rowlands method were developed (Rodgers and Rowland, 2006) (). The contribution of microsomal metabolism and renal and biliary excretion to the clearance was added to the PBPK models when applicable (Fig. 1; Table 1).

Aldehyde oxidase is present in organs other than the liver (Moriwaki et al., 2001; Nishimura and Naito, 2006). To study the potential impact of extrahepatic metabolism, the activity per $\mathrm{mg}$ of cytosolic protein in the kidney was assumed to be the same as that of the liver, and the free intrinsic activity was scaled based on the human cytosolic protein per gram of kidney, kidney weight, and blood flow. A cytosolic protein per gram of kidney value of $40.6 \mathrm{mg} / \mathrm{g}$ was used (Scotcher, 2016). Similarly, lung metabolism was also explored, and the activity per mg of cytosolic protein was assumed to be the same as that of the liver. The IVIVE scaling approach (Fig. 2) using the well-stirred lung model (Yang, 2007) is integrated within the Simcyp Simulator and was simply entered as additional lung clearance; it was calculated with the following scaling parameters: cytosolic protein per gram of lung yield of $20 \mathrm{mg} / \mathrm{g}$, a lung tissue weight (excluding blood) of $550 \mathrm{~g}$, and a cardiac output of $386 \mathrm{l} / \mathrm{h}$. Cytosolic protein per gram of lung was obtained using the $\mathrm{S} 9$ fraction in the lung of $28 \mathrm{mg} / \mathrm{g}$ tissue (Kozminski et al., 2019) and by assuming that the fraction of cytosolic protein to S9 protein is constant between the lung, liver, and kidney. Because of the limited expression of AO in the intestine (Moriwaki et al., 2001; Nishimura and Naito, 2006; Hutzler et al., 2012), intestinal metabolism was not considered in the current analysis.

A linear regression between the predicted $\mathrm{CL}_{\mathrm{IV}}$ (dose/AUC $\mathrm{A}_{\text {-infinity }}$ after a simulated single intravenous dose) and the observed $\mathrm{CL}_{\mathrm{IV}}$ was calculated using $\mathrm{R}$ (version 3.5.1, www.r-project.org) with a weighting option of $1 / \mathrm{Y}_{-}$pred to avoid bias toward the highest clearance value.

A sensitivity analysis was done on the $\mathrm{AO}$ intrinsic clearance of the six drugs to explore the impact of increasing the intrinsic clearance in the liver and in extrahepatic organs on the predicted $\mathrm{CL}_{\mathrm{IV}}$. In the sensitivity analyses described here, the kidney was used as a surrogate organ to account for all the extrahepatic metabolism in the body. The kidney was chosen as the site of extrahepatic metabolism for practical reasons rather than splitting the clearance over several different organs. The ratio between the observed and predicted $\mathrm{CL}_{\mathrm{IV}}$ was calculated.

The intrinsic clearance of each compound was then optimized using available PK profiles except for BIBX1382, for which no concentration-time profile was available and only the clearance is reported in the literature. The following dosing regimens were used to simulate the PK profiles: $\mathrm{O}^{6}$-benzylguanine-bolus administration of $20 \mathrm{mg} / \mathrm{m}^{2}$ to seven patients with cancer aged $45-74$ years (prop. of female $=0.42)$ (Tserng et al., 2003); carbazeran-10-minute infusion of $1.28 \mathrm{mg} / \mathrm{kg}$ of carbazeran to seven healthy male volunteers aged $20-50$ years (Kaye et al., 1984); zaleplon-30-minute infusion of $5 \mathrm{mg}$ to 10 healthy subjects aged 30-32 years (prop. of female $=0.5$ ) (Rosen et al., 1999); ziprasidone-1hour infusion of $5 \mathrm{mg}$ to 13 male subjects aged 19-37 years (Miceli et al., 2005); and zoniporide-1-hour infusion of $80 \mathrm{mg}$ to four male healthy subjects aged 18-55 years (Dalvie et al., 2010). Additionally, the Kp scalar was optimized for carbazeran $(=0.13)$ and zoniporide $(=0.45)$ to better fit the observed volume of distribution at steady state $\left(\mathrm{V}_{\mathrm{ss}}\right)$. The required (in silico) intrinsic clearance from the PBPK model was then compared with the measured (in vitro) intrinsic clearance to calculate a scaling factor for each compound.

Additionally, to verify the usefulness of this approach in predicting clearance of a new compound, an average scaling factor was applied to the in vitro intrinsic clearance of the studied drugs. The average scaling factor was calculated based on all the drugs except the one that was being predicted.

\section{Results}

The investigated compounds covered a wide range of $\mathrm{fm}_{\mathrm{AO}}$, ranging from 0.064 (ziprasidone) to 0.98 (carbazeran). The $\log P$ values ranged from 1.04 ( $\mathrm{O}^{6}$-benzylguanine) to 3.97 (BIBX1382), and there was one neutral compound, four basic compounds, and an ampholyte. Table 1 summarizes the physicochemical data and the measured $\mathrm{CL}_{\text {int,u }}$ (free intrinsic clearance) values obtained in HLC, HLS9, and HLM. The results

TABLE 2

Comparison of intrinsic clearance data obtained for AO from literature reports, in-house measurements, and retrograde scaling Laboratory-specific scaling factors for six AO substrates.

\begin{tabular}{|c|c|c|c|c|c|c|}
\hline $\begin{array}{l}\mathrm{HLC} \mathrm{CL}_{\text {int,u }} \\
(\mu \mathrm{l} / \mathrm{min} \text { per mg } \\
\text { Cytosolic Protein) }\end{array}$ & $\mathrm{O}^{6}$-Benzylguanine & BIBX1382 & Carbazeran & Zaleplon & Ziprasidone & Zoniporide \\
\hline Literature & $\begin{array}{l}16.52 \text { (Roy et al., 1995; } \\
\text { Zientek et al., 2010) }\end{array}$ & $\begin{array}{l}860.7 \text { (Hutzler } \\
\text { et al., 2012) }\end{array}$ & $\begin{array}{l}446 \text { (Zientek et al., } \\
\text { 2010; Fu et al., 2013) }\end{array}$ & $\begin{array}{l}3.9 \text { (Lake et al., 2002; } \\
\text { Zientek et al., 2010) }\end{array}$ & $\begin{array}{l}410.3 \text { (Obach } \\
\text { et al., 2012) }\end{array}$ & $\begin{array}{c}41.2 \text { (Dalvie et al., 2010; } \\
\text { Zientek et al., 2010; Fu et al., } \\
\text { 2013) }\end{array}$ \\
\hline $\begin{array}{l}\text { Measured } \\
\quad \text { (this study) }\end{array}$ & 10.9 & 1062 & 175 & 1.86 & 33.4 & 13.1 \\
\hline $\begin{array}{l}\text { Estimated } \\
\quad{\text { (this study })^{a}}^{a}\end{array}$ & 109.4 & 3717 & 764 & 22.34 & 3338 & 33 \\
\hline $\begin{array}{l}\text { Scaling factor (this } \\
\text { study) }\end{array}$ & 10 & 3.5 & 4.35 & 12 & 100 & 2.5 \\
\hline
\end{tabular}

${ }^{a}$ Estimated based on the PK profiles or reported CL. 

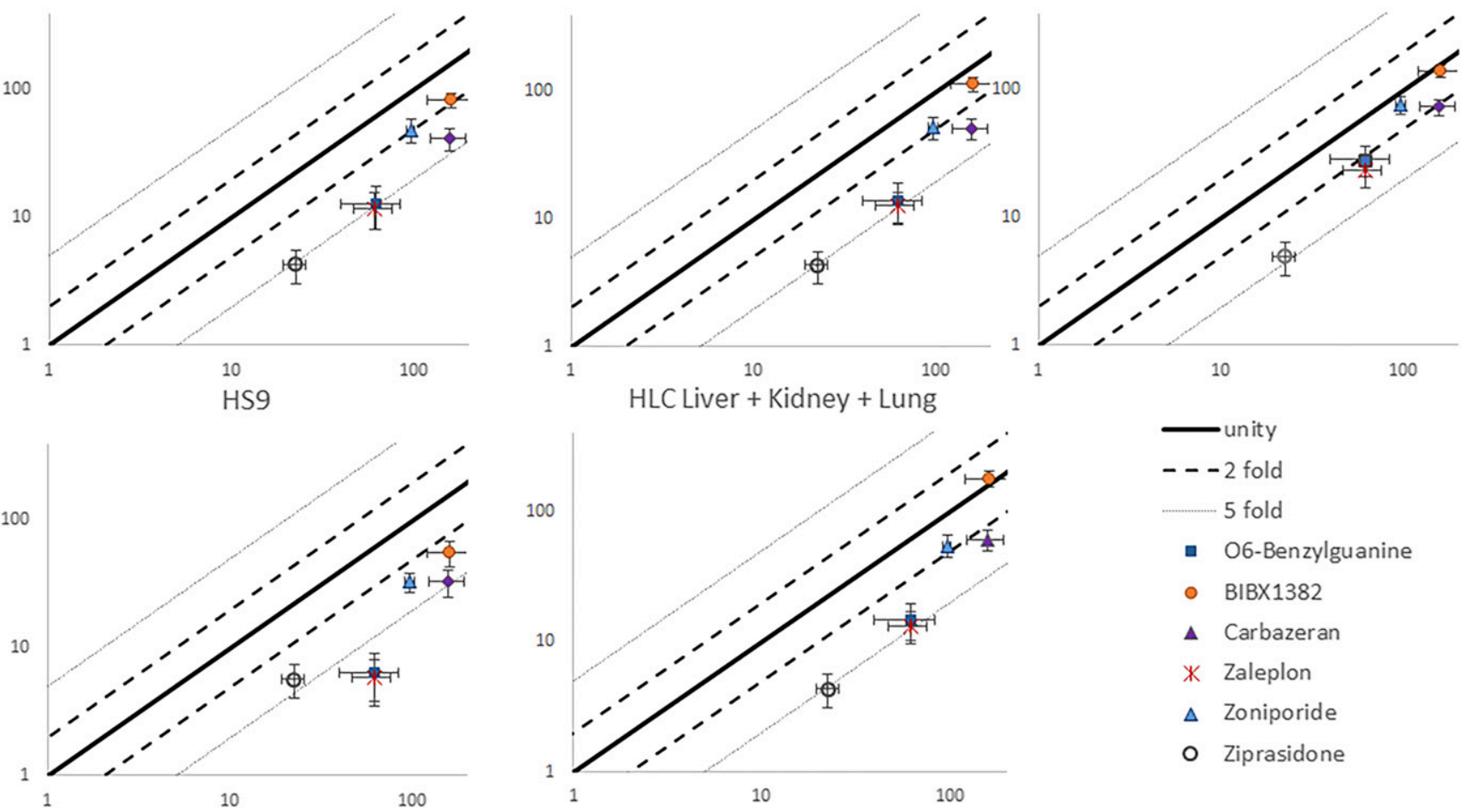

Fig. 3. Predicted $\mathrm{CL}_{\mathrm{IV}}\left( \pm\right.$ S.D.) compared with mean observed $\mathrm{CL}_{\mathrm{IV}}$ ( \pm S.D.). Results are compared with the unity line and 2- and 5-fold bias lines. HLS9, human liver $\mathrm{S} 9$; REF, reference.

were overall in good agreement with literature data except for ziprasidone, in which the HLC $\mathrm{CL}_{\text {int,u }}$ was more than 10-fold lower than previously reported values [33.4 vs. $410.3 \mu 1 / \mathrm{min}$ per mg (Obach et al., 2012)] (Table 2).

Prediction of Intravenous Clearance Using PBPK Models. Figure 3 compares the observed $\mathrm{CL}_{\mathrm{IV}}$ to the $\mathrm{CL}_{\mathrm{IV}}$ predicted from the PBPK model (Table 1).

HLC Liver Only. Using HLC data, the average extent of underprediction of $\mathrm{CL}_{\mathrm{IV}}$ was 3.8 and ranged from 1.9 to 5.2 (Fig. 3). The best prediction was obtained for BIBX1382 (1.9-fold), and the biggest difference was for ziprasidone (5.2-fold). A coefficient of 2.77 was obtained using a weighted linear regression between the observed clearance and predicted clearance. For all the compounds, the average CV\% for CL predicted by the PBPK models was 26\% (range: $15.9 \%-37.5 \%)$. The predicted variability was in accordance with the mean observed CV of $21 \%$ (range: $5.7 \%-35.9 \%$ ). Fig. 4 shows that no trend between the extent of underestimation and the predicted fm by AO could be observed.

HLS9 Liver Only. Using HLS9 experiment, the $\mathrm{CL}_{\mathrm{IV}}$ was predicted to be lower than the $\mathrm{CL}_{\mathrm{IV}}$ obtained from HLC with an average extent of underprediction of 5.8, and it ranged from 2.9 (BIBX1382) to 10.6 (Zaleplon) (Fig. 3). A coefficient of 4.02 was obtained using a weighted linear regression between the observed clearance and predicted clearance. Simcyp workspaces for these six compounds using HLS9 data are provided as Supplemental Data Sets 1-6.

HLC Liver and Kidney. When the metabolism in the liver and the kidney were included (assuming the same intrinsic clearance per mg of cytosolic protein), a small improvement of the prediction of the $\mathrm{CL}_{\mathrm{IV}}$ was observed. The extent of underprediction of $\mathrm{CL}_{\mathrm{IV}}$ was 3.5 and ranged from 1.4 (BIBX1382) to 5.2 (ziprasidone) (Fig. 3). A coefficient of 2.26 was obtained using a weighted linear regression between the observed clearance and predicted clearance.
HLC Liver, Kidney, and Lung. When the metabolism in the liver, the kidney, and the lung were included (assuming the same activity per $\mathrm{mg}$ of cytosolic protein), a more pronounced increase in the accuracy of prediction of the $\mathrm{CL}_{\mathrm{IV}}$ was observed. The average extent of underprediction of $\mathrm{CL}_{\mathrm{IV}}$ was 3.2 and ranged from 0.9 (BIBX1382) to 5.2 (ziprasidone) (Fig. 3). A coefficient of 1.74 was obtained using a weighted linear regression between the observed clearance and predicted clearance.

Sensitivity Analyses. Figure 5 shows the sensitivity analyses made on a scaling factor applied to the intrinsic clearance and considering the metabolism in liver and kidney. $\mathrm{O}^{6}$-benzylguanine and zaleplon have similar profiles and reach the observed $\mathrm{CL}_{\mathrm{IV}}$ with a scaling factor of around 15; BIXB1382 quickly reaches a clearance close to the observed clearance but also becomes quickly nonsensitive to any changes in $\mathrm{CL}_{\text {int,u }}$. Carbazeran reaches a plateau around $75 \%$ of the observed $\mathrm{CL}_{\mathrm{IV}}$, and the blood flow is the limiting factor in this case. Ziprasidone $\mathrm{CL}_{\mathrm{int}, \mathrm{u}}$ was a significantly lower value than reported in the literature (Table 2),

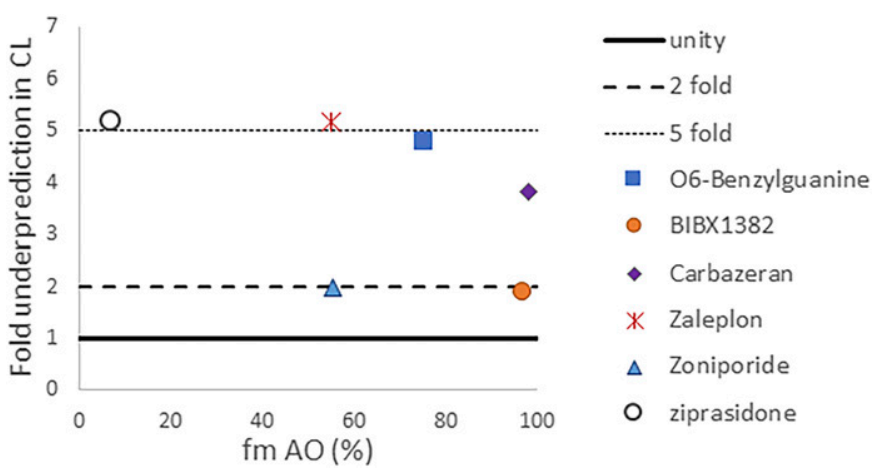

Fig. 4. Fold underprediction of clearance when considering only hepatic metabolism compared with the predicted $\mathrm{fm}_{\mathrm{AO}}$. 


\section{Sensitivity analyses}

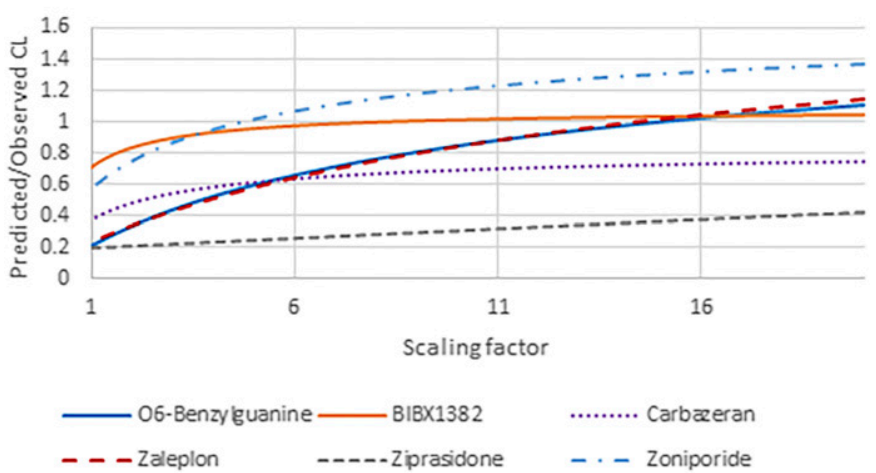

Fig. 5. Sensitivity analyses on the intrinsic activity of AO in HLC assuming metabolism in the liver and the kidney.

and even with a 20-fold increase in the intrinsic clearance the observed $\mathrm{CL}_{\mathrm{IV}}$ is not attained. Zoniporide $\mathrm{CL}_{\mathrm{IV}}$ reaches the observed $\mathrm{CL}_{\mathrm{IV}}$ with a scaling factor of 3.5 .

PK Profiles. Figure 6 shows the simulated profiles with and without optimization compared with the observed $\mathrm{CL}_{\mathrm{IV}}$. The intrinsic clearance $\left(\mathrm{CL}_{\mathrm{int}} \mathrm{AO}\right)$ and scaling factor obtained are reported in Table 2 . In this study, an average $\mathrm{CL}_{\text {int, }}$ AO scaling factor of 6.5 was necessary to recover the $\mathrm{PK}$ profiles. Ziprasidone has a low $\mathrm{fm}_{\mathrm{AO}}$, and the metabolic activity was lower in this study than previously reported, and for this reason ziprasidone was excluded from the calculation of the average scalar analysis.

Table 3 shows that using the average scalar factor from the other drugs significantly improves the prediction of $\mathrm{CL}_{\mathrm{IV}}$, with an average underprediction of 1.5 (range 0.98-1.96). All drugs were predicted within a 2-fold error, which is considered adequate for predictions early in drug discovery. Applying the scaling factor of 6.5 to ziprasidone, the $\mathrm{CL}_{\mathrm{IV}}$ was underpredicted by 3.9 -fold, increasing the average fold underprediction to 1.9 for the six compounds.

\section{Discussion}

Using IVIVE approaches to predict aldehyde oxidase-mediated clearance has typically resulted in a significant underprediction of the observed clearance, resulting in the clinical failure of multiple drugs that are metabolized by AO (Fan et al., 2016; Jensen et al., 2017). This study explored IVIVE of $\mathrm{CL}_{\mathrm{AO}}$ and aimed to develop a methodology to aid informed decision making on newly developed drug candidates that showed potential to be metabolized by $\mathrm{AO}$, and a workflow for AOmediated clearance prediction is proposed. Overall, using HLC, the intravenous CL continued to be underestimated, but HLC performed better than HLS9 in this study. Ideally the in vitro system of each laboratory should be characterized by measuring the $\mathrm{CL}_{\mathrm{int}, \mathrm{u}}$ of the selected probe substrates present in this study, and then a scaling factor should be calculated using the information in Table 2 and applied on the intrinsic clearance assuming metabolism in the liver and the kidney as explained in the Materials and Methods section. The coefficient obtained with the linear regression could also be applied as an empirical scalar directly on the $\mathrm{CL}_{\mathrm{IV}}$. If in-house probe substrate $\mathrm{CL}_{\text {int }}$ data are not available, a scalar of 4.6 could be applied on the $\mathrm{CL}_{\text {int,u }}$ based on the literature-published values of AOmediated metabolism.

Measured B/P and fu were like values reported in the literature (Alousi et al., 2007; Zientek et al., 2010; Akabane et al., 2012). The average in vitro
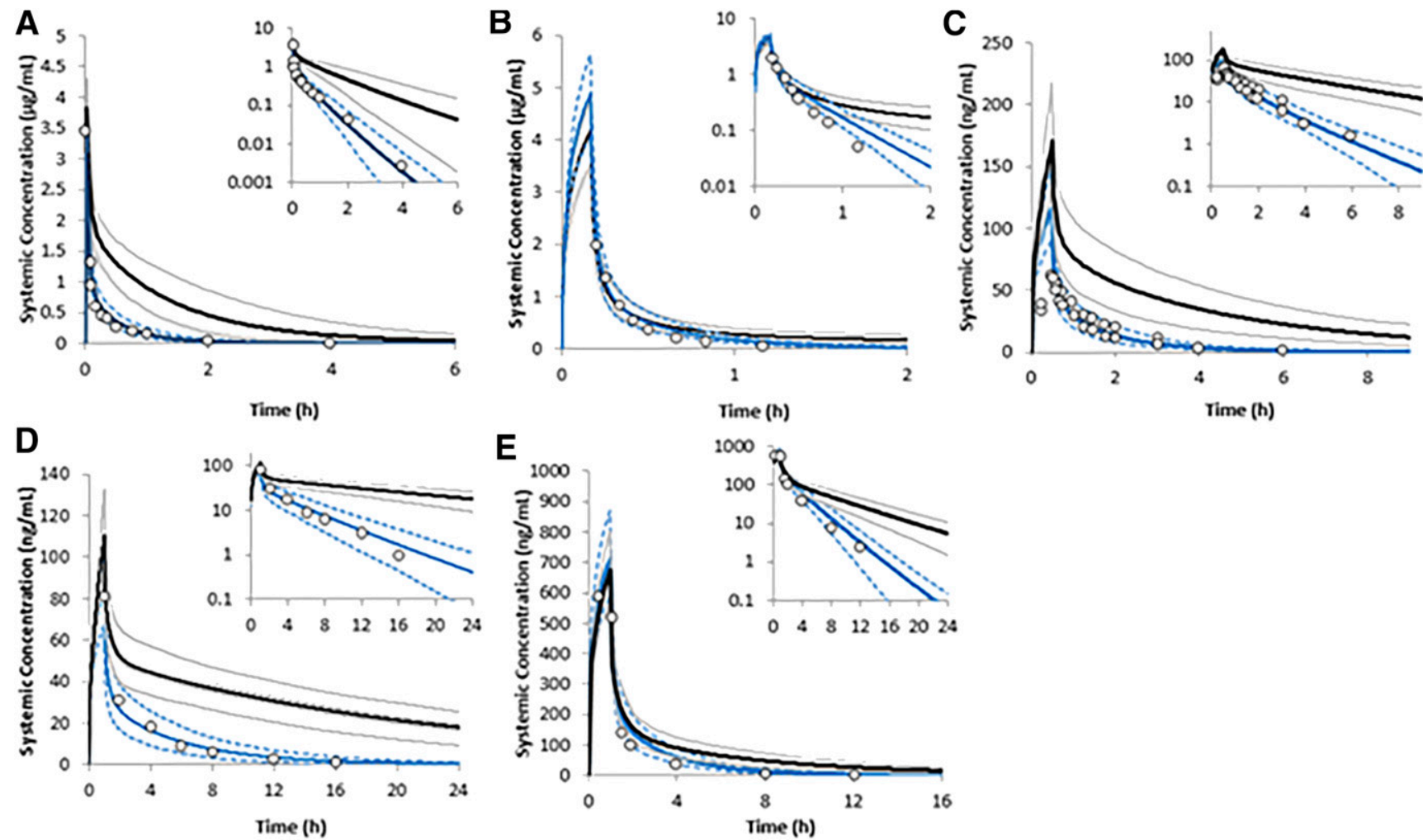

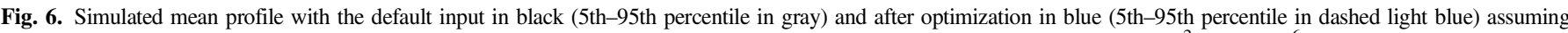

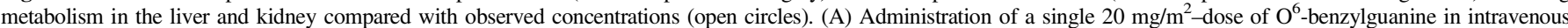

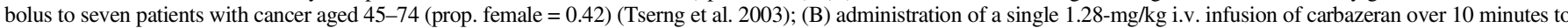

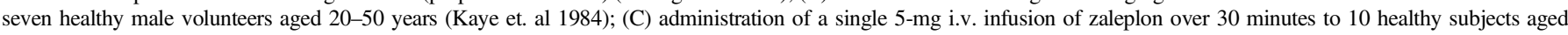

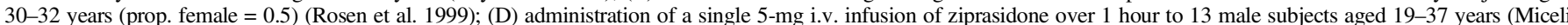
et al. 2005); (E) administration of a single 80-mg i.v. infusion of zoniporide over 1 hour to four male healthy subjects aged 18-55 years (Dalvie et al. 2010). 
TABLE 3

Use of scaling factor from other compounds to predict the CL

The individual scaling factors are noted below the compounds; to predict the CL of a drug, the avg. scaling factor of the other drugs was used [i.e., the scalar used for 06-benzylguanine was 5.6 (avg. of $3.5,4.35,12$, and 2.5$)]$.

\begin{tabular}{|c|c|c|c|c|c|c|c|c|c|}
\hline Ref & $\mathrm{O}^{6}$-Benzylguanine & BIBX1382 & Carbazeran & Zaleplon & Zoniporide & $\begin{array}{l}\text { Avg. Scaling } \\
\text { Factor }\end{array}$ & Observed CL & Predicted CL & Ratio \\
\hline 06-Benzylguanine ${ }^{\mathrm{a}}$ & $\mathrm{x}$ & 3.5 & 4.35 & 12 & 2.5 & 5.6 & $62 \pm 22$ & $40 \pm 9$ & 1.54 \\
\hline BIBX1382 & 10 & $\mathrm{x}$ & 4.35 & 12 & 2.5 & 7.2 & $162 \pm 42$ & $154 \pm 18$ & 1.05 \\
\hline Carbazeran & 10 & 3.5 & $\mathrm{X}$ & 12 & 2.5 & 7.0 & $158 \pm 35$ & $88 \pm 11$ & 1.79 \\
\hline Zaleplon & 10 & 3.5 & 4.35 & $\mathrm{x}$ & 2.5 & 5.1 & $61 \pm 15$ & $32 \pm 7$ & 1.96 \\
\hline Zoniporide & 10 & 3.5 & 4.35 & 12 & $\mathrm{x}$ & 7.5 & $96 \pm 6$ & $98 \pm 13$ & 0.98 \\
\hline
\end{tabular}

${ }^{a}$ AUC $(0-4$ h) to match the reported AUC; Ref, reference.

intrinsic clearances obtained from the literature were overall higher, especially for ziprasidone. One reason for this discrepancy in in vitro intrinsic clearances could be explained by the variability of activity in AO across human liver cytosolic fractions (Hutzler et al., 2014) or, alternatively, due to the nature of the experimental protocol (i.e., incubation and sampling time, buffer). It is possible that human donor liver tissues that were preserved in allopurinol-containing University of Wisconsin solution may exhibit weak AO inhibitory effect at higher concentrations and could affect the scaling factors calculated in this study. Nevertheless, Barr et al. (2014) have shown that small residual amounts of allopurinol or oxypurinol did not appear to impact AO activity. By analyzing the different protocols used in the literature, no clear association between experimental condition and measured $\mathrm{CL}_{\text {int }}$ was observed, and a larger data set or new specific in vitro assays looking at the impact of the protocol would be required (Dick, 2018).

In this data set, the fraction metabolized by AO was estimated by including all known pathways; however, even if some inhibitors of aldehyde oxidase have been identified in vitro (Johns, 1967; Johnson et al., 1985; Obach, 2004), limited clinical drug interactions via inhibition of aldehyde oxidase have been recorded, thus restricting the additional validation of in vivo probe substrates or inhibitors for the verification of the $\mathrm{fm}_{\mathrm{AO}}$ of given substrate.

Some compounds have an intravenous clearance higher than the hepatic blood flow, suggesting extrahepatic elimination. Aldehyde oxidase is expressed in multiple tissues, including the kidney (Moriwaki et al., 2001; Nishimura and Naito, 2006). A scenario assuming an AO activity/ expression per mg of cytosolic protein in the kidney identical to that in the liver was simulated in the PBPK models, and even though an improved prediction was observed, the underprediction of CL was still significant. Expression of $\mathrm{AO}$ in the intestine is limited, and incubations of $\mathrm{AO}$ substrate with human intestinal cytosol resulted in no measurable metabolism (Moriwaki et al., 2001; Nishimura and Naito, 2006; Hutzler et al., 2012); therefore, intestinal metabolism was not considered in the current analysis. The lung is a highly perfused organ with a significant tissue volume and an AO absolute abundance within 6-fold of that in the liver (Ezkurdia et al., 2015). Previous studies have attempted to incorporate lung metabolism into IVIVE approaches for AO-mediated clearance (Kozminski et al., 2019); however, in this study the reported intrinsic activity in the lung was almost 1000-fold lower than that in the liver, explaining the absence of significant impact of lung metabolism on the overall predicted clearance (Kozminski et al., 2019). In this study, even assuming a tissue activity per mg of lung cytosol identical to that in the liver did not explain the underestimation of clearance observed for all compounds (Fig. 3).

Aldehyde oxidase has been shown to have a limited stability, and freeze-thaw cycles might result in higher variability, and therefore, the metabolic activity might be underestimated in in vitro assays (Sherratt and Damani, 1989; Hutzler et al., 2012). The underestimation of AO clearance is likely to be due to an underestimation of the intrinsic clearance as well as extrahepatic metabolism.
An additional way to scale from in vitro to in vivo would be to use the absolute abundance rather than activity per $\mathrm{mg}$ of protein. This approach would allow the use of recombinant AO and, therefore, have an extremely specific system with less risk of contamination from other enzymes (i.e., xanthine oxidase). In addition, the absolute abundance in all of the different tissues in the body could be accounted for with a single in vitro metabolism measurement. So far, the absolute abundance in the liver has been measured (Barr et al., 2013; Fu et al., 2013; Ezkurdia et al., 2015; Wiśniewski et al., 2016), and recombinant AOs are available. Unfortunately, there is a lot of variability between the different laboratories (mean: $34.5 \mathrm{pmol} / \mathrm{mg}$ of cytosolic protein; range: $1.41-60.2 \mathrm{pmol} / \mathrm{mg}$ of cytosolic protein, four studies, a total of 30 livers), and the absolute abundance has not been measured in the recombinant systems.

An additional aim of this work was to gather the input information for PBPK models for the different AO substrate compounds so that the models could be available for use in future research efforts. The PBPK models could be used to investigate different aspects, such as interindividual differences in $\mathrm{AO}$ expression; to study the interaction between AO substrates and inhibitors [e.g., between zaleplon and cimetidine (Dalvie and Di, 2019)]; or to investigate the PK of these compounds in different populations of individuals.

\section{Conclusion}

A workflow for NCE metabolized by AO was suggested, and an empirical scaling factor of three on the predicted $\mathrm{CL}_{\mathrm{IV}}$ based on HLC data could be applied for NCEs that are significantly metabolized by AO when using PBPK models for predicting the exposure of NCEs in the human. Alternatively, a scaling factor of 6.5 could be applied to the AO intrinsic clearance in the liver and kidney. Ideally each laboratory should develop a correlation using a set of probe substrates under their own assay conditions; however, if the in vitro $\mathrm{CL}_{\mathrm{int}, \mathrm{u}}$ values for probe substrates are not available in a given laboratory, an empirical scaling factor of 4.6 based on this work could be applied for $\mathrm{CL}_{\mathrm{int}, \mathrm{u}}$ in HLC. Additional research on the impact of the in vitro study designs and extrahepatic metabolism is suggested to understand the mechanism behind the systematic underprediction observed for AO.

\section{Authorship Contributions}

Participated in research design: De Sousa Mendes, Gardner, Neuhoff, Pilla Reddy.

Conducted experiments: Orton, Jones.

Performed data analysis: De Sousa Mendes, Pilla Reddy.

Wrote or contributed to the writing of the manuscript: De Sousa Mendes, Orton, Humphries, Jones, Gardner, Neuhoff, Pilla Reddy.

\section{References}

Akabane T, Gerst N, Masters JN, and Tamura K (2012) A quantitative approach to hepatic clearance prediction of metabolism by aldehyde oxidase using custom pooled hepatocytes. Xenobiotica 42:863-871. 
Alousi AM, Boinpally R, Wiegand R, Parchment R, Gadgeel S, Heilbrun LK, Wozniak AJ, DeLuca P, and LoRusso PM (2007) A phase 1 trial of XK469: toxicity profile of a selective topoisomerase IIbeta inhibitor. Invest New Drugs 25:147-154.

Al salhen KS (2014) In vitro oxidation of aldehyde oxidase from rabbit liver: specificity toward endogenous substrates. Journal of King Saud University-Science 26:67-74.

Austin RP, Barton P, Cockroft SL, Wenlock MC, and Riley RJ (2002) The influence of nonspecific microsomal binding on apparent intrinsic clearance, and its prediction from physicochemical properties. Drug Metab Dispos 30:1497-1503.

Barr, Choughule, Nepal, et al. (2014) Barr JT, Choughule KV, Nepal S, Wong T, Chaudhry AS Joswig-Jones CA, Zientek M, Strom SC, Schuetz EG, Thummel KE, et al. (2014) Drug Metab Dispos 42:695-699. Why do most human liver cytosol preparations lack xanthine oxidase activity? 42:695-699 PMC4109211.

Barr JT, Jones JP, Joswig-Jones CA, and Rock DA (2013) Absolute quantification of aldehyde oxidase protein in human liver using liquid chromatography-tandem mass spectrometry. Mol Pharm 10:3842-3849.

Cubitt HE (2009) In vitro assessment of hepatic and intestinal conjugation reactions and impact on drug clearance prediction, School of Pharmacy and Pharmaceutical Sciences, University of Manchester.

Dalvie D and Di L (2019) Aldehyde oxidase and its role as a drug metabolizing enzyme. Pharmacol Ther 201:137-180.

Dalvie D, Xiang C, Kang P, and Zhou S (2013) Interspecies variation in the metabolism of zoniporide by aldehyde oxidase. Xenobiotica 43:399-408.

Dalvie D, Zhang C, Chen W, Smolarek T, Obach RS, and Loi C-M (2010) Cross-species comparison of the metabolism and excretion of zoniporide: contribution of aldehyde oxidase to interspecies differences. Drug Metab Dispos 38:641-654.

Dick RA (2018) Refinement of in vitro methods for identification of aldehyde oxidase substrates reveals metabolites of kinase inhibitors. Drug Metab Dispos 46:846-859.

Dolan ME, Roy SK, Fasanmade AA, Paras PR, Schilsky RL, and Ratain MJ (1998) O6benzylguanine in humans: metabolic, pharmacokinetic, and pharmacodynamic findings. $J$ Clin Oncol 16:1803-1810.

Ezkurdia I, Calvo E, Del Pozo A, Vázquez J, Valencia A, and Tress ML (2015) The potential clinical impact of the release of two drafts of the human proteome. Expert Rev Proteomics 12 579-593.

Fan PW, Zhang D, Halladay JS, Driscoll JP, and Khojasteh SC (2016) Going beyond common drug metabolizing enzymes: case studies of biotransformation involving aldehyde oxidase, $\gamma$-glutamyl transpeptidase, cathepsin B, flavin-containing monooxygenase, and ADP-ribosyltransferase Drug Metab Dispos 44:1253-1261.

Fu C, Di L, Han X, Soderstrom C, Snyder M, Troutman MD, Obach RS, and Zhang H (2013) Aldehyde oxidase 1 (AOX1) in human liver cytosols: quantitative characterization of AOX1 expression level and activity relationship. Drug Metab Dispos 41:1797-1804.

Hutzler JM, Yang Y-S, Albaugh D, Fullenwider CL, Schmenk J, and Fisher MB (2012) Characterization of aldehyde oxidase enzyme activity in cryopreserved human hepatocytes. Drug Metab Dispos 40:267-275.

Hutzler JM, Yang Y-S, Brown C, Heyward S, and Moeller T (2014) Aldehyde oxidase activity in donor-matched fresh and cryopreserved human hepatocytes and assessment of variability in 75 donors. Drug Metab Dispos 42:1090-1097.

Jensen KG, Jacobsen A-M, Bundgaard C, Nilausen DØ, Thale Z, Chandrasena G, and Jørgensen M (2017) Lack of exposure in a first-in-man study due to aldehyde oxidase metabolism: in vestigated by use of $14 \mathrm{C}$-microdose, humanized mice, monkey pharmacokinetics, and in vitro methods. Drug Metab Dispos 45:68-75.

Johns DG (1967) Human liver aldehyde oxidase: differential inhibition of oxidation of charged and uncharged substrates. $J$ Clin Invest 46:1492-1505.

Johnson C, Stubley-Beedham C, and Stell JG (1985) Hydralazine: a potent inhibitor of aldehyde oxidase activity in vitro and in vivo. Biochem Pharmacol 34:4251-4256.

Kaye B, Offerman JL, Reid JL, Elliott HL, and Hillis WS (1984) A species difference in the presystemic metabolism of carbazeran in dog and man. Xenobiotica 14:935-945.

Kozminski KD, Heyward S, and Zientek M (2019) Aldehyde oxidase activity in human vascular tissue and its potential contribution to extra-hepatic metabolism. Drug Metab Pharmacokinet 34 S62-S63.

Lake BG, Ball SE, Kao J, Renwick AB, Price RJ, and Scatina JA (2002) Metabolism of zaleplon by human liver: evidence for involvement of aldehyde oxidase. Xenobiotica 32:835-847.
Liu X, Wang J-Q, and Zheng Q-H (2005) Lipophilicity coefficients of potential tumor imaging agents, positron-labeled O(6)-benzylguanine derivatives. Biomed Chromatogr 19:379-384.

Miceli JJ, Wilner KD, Swan SK, and Tensfeldt TG (2005) Pharmacokinetics, safety, and tolerability of intramuscular ziprasidone in healthy volunteers. J Clin Pharmacol 45:620-630.

Montefiori M, Jørgensen FS, and Olsen L (2017) Aldehyde oxidase: reaction mechanism and prediction of site of metabolism. ACS Omega 2:4237-4244.

Moriwaki Y, Yamamoto T, Takahashi S, Tsutsumi Z, and Hada T (2001) Widespread cellular distribution of aldehyde oxidase in human tissues found by immunohistochemistry staining. Histol Histopathol 16:745-753.

Nishimura M and Naito S (2006) Tissue-specific mRNA expression profiles of human phase I metabolizing enzymes except for cytochrome P450 and phase II metabolizing enzymes. Drug Metab Pharmacokinet 21:357-374.

Obach RS (2004) Potent inhibition of human liver aldehyde oxidase by raloxifene. Drug Metab Dispos 32:89-97.

Obach RS (2011) Predicting clearance in humans from in vitro data. Curr Top Med Chem 11 334-339.

Obach RS, Prakash C, and Kamel AM (2012) Reduction and methylation of ziprasidone by glutathione, aldehyde oxidase, and thiol S-methyltransferase in humans: an in vitro study. Xenobiotica 42:1049-1057.

Renwick AB, Ball SE, Tredger JM, Price RJ, Walters DG, Kao J, Scatina JA, and Lake BG (2002) Inhibition of zaleplon metabolism by cimetidine in the human liver: in vitro studies with subcellular fractions and precision-cut liver slices. Xenobiotica 32:849-862.

Rodgers Trudy and Rowland Malcolm (2006) Physiologically based pharmacokinetic modelling 2 predicting the tissue distribution of acids, very weak bases, neutrals and zwitterions. J Pharm Sci 95 (6): 1238-1257, doi: 10.1002/jps.20502 16639716

Rosen AS, Fournié P, Darwish M, Danjou P, and Troy SM (1999) Zaleplon pharmacokinetics and absolute bioavailability. Biopharm Drug Dispos 20:171-175.

Roy SK, Korzekwa KR, Gonzalez FJ, Moschel RC, and Dolan ME (1995) Human liver oxidative metabolism of O6-benzylguanine. Biochem Pharmacol 50:1385-1389.

Scotcher D (2016) Physiological scaling factors and Mechanistic models for prediction of Renal clearance from in vitro data, Faculty of Medical and Human Sciences Manchester, University of Manchester.

Sherratt AJ and Damani LA (1989) Activities of cytosolic and microsomal drug oxidases of rat hepatocytes in primary culture. Drug Metab Dispos 17:20-25.

Tanoue C, Sugihara K, Uramaru N, Watanabe Y, Tayama Y, Ohta S, and Kitamura S (2013) Strain difference of oxidative metabolism of the sedative-hypnotic zaleplon by aldehyde oxidase and cytochrome P450 in vivo and in vitro in rats. Drug Metab Pharmacokinet 28:269-273.

Tracey WR, Allen MC, Frazier DE, Fossa AA, Johnson CG, Marala RB, Knight DR, and GuzmanPerez A (2003) Zoniporide: a potent and selective inhibitor of the human sodium-hydrogen exchanger isoform 1 (NHE-1). Cardiovasc Drug Rev 21:17-32.

Tserng K-Y, Ingalls ST, Boczko EM, Spiro TP, Li X, Majka S, Gerson SL, Willson JK, and Hoppel CL (2003) Pharmacokinetics of O6-benzylguanine (NSC637037) and its metabolite, 8-oxo-O6-benzylguanine. J Clin Pharmacol 43:881-893.

Wiśniewski JR, Wegler C, and Artursson P (2016) Subcellular fractionation of human liver reveals limits in global proteomic quantification from isolated fractions. Anal Biochem 509:82-88

Yang Jiansong, Masoud Jamei, and Karen Yeo (2007) Misuse of the Well-Stirred Model of Hepatic Drug Clearance. Drug Metabolism and Disposition 35:501-502.

Zientek M, Jiang Y, Youdim K, and Obach RS (2010) In vitro-in vivo correlation for intrinsic clearance for drugs metabolized by human aldehyde oxidase. Drug Metab Dispos 38 1322-1327.

Zientek MA and Youdim K (2015) Reaction phenotyping: advances in the experimental strategies used to characterize the contribution of drug-metabolizing enzymes. Drug Metab Dispos $\mathbf{4 3}$ $163-181$.

Address correspondence to: Dr. Venkatesh Pilla Reddy, Modelling and Simulation, Research \& Early Development, AstraZeneca, Hodgkin Bldg. c/o B310, Cambridge Science Park, Milton Rd., Cambridge, Cambridgeshire CB4 oWG, UK. E-mail: venkatesh.reddy@astrazeneca.com 